\title{
Mexikanische Cycadeen und deren Schutzsammlung im Botanischen Garten von Xalapa, Veracruz, Mexiko
}

\author{
Julio Valentin Schneider \& Marleen Mika
}

\begin{abstract}
Cycads are among the most threatened plants on earth. Here, aspects of the morphology, systematics, biogeography, uses, and conservation of cycads are reviewed with emphasis on the Mexican species. Additionally, the Botanic Garden of Xalapa, Mexico, and its cycads conservation program are presented.
\end{abstract}

\section{Zusammenfassung}

Cycadeen stellen eine der am stärksten bedrohten Pflanzengruppen weltweit dar. Es werden Aspekte der Morphologie, Systematik, Biogeografie, des Nutzens und Artenschutzes der Cycadeen behandelt. Außerdem werden der Botanische Garten Xalapa, Mexiko, und seine Cycadeen-Schutzprogamme vorgestellt.

\section{Cycadeen - ein Überblick}

Die Cycadeen oder Palmfarne sind nicht nur attraktive Zierpflanzen, sondern auch eine botanisch außergewöhnlich interessante Gruppe innerhalb der Samenpflanzen, leider aber auch stark gefährdet. Wie der deutsche Name schon sagt, erinnern die Palmfarne mit ihren teils hochwüchsigen, unverzweigten Stämmen und den an der Sprossspitze sich schirmförmig ausbreitenden, farnwedelartigen Blättern an Palmen oder (Baum-)Farne (Abb. 1). Treten noch die ungewöhnlich anmutenden zapfenförmigen Blütenstände hinzu, entsteht unweigerlich der Eindruck, dass es sich hierbei um besonders urwüchsige Pflanzen handelt. Und in der Tat gelten sie als lebende Fossilien. Erste Nachweise der Cycadeen datieren aus dem Perm und weisen ein Alter von 250-280 Millionen Jahren auf (Gao \& Thomas 1989, Norstog \& Nicholls 1997). Damit stellen sie die älteste Kronengruppe der Samenpflanzen dar (Hermsen et al. 2006). Stammesgeschichtlich betrachtet gehören sie zu den Nacktsamern, allerdings konnten die Verwandtschaftsverhältnisse innerhalb dieser Gruppe trotz mehrerer moderner molekularsystematischer Stu-

Abb. 1: Microcycas calocoma, ein Endemit Kubas mit einer an Palmen erinnernden Wuchsform. Hier ungewöhnlicherweise mit verzweigtem Stamm, vermutlich bedingt durch eine Beschädigung der Hauptachse in jungen Jahren.

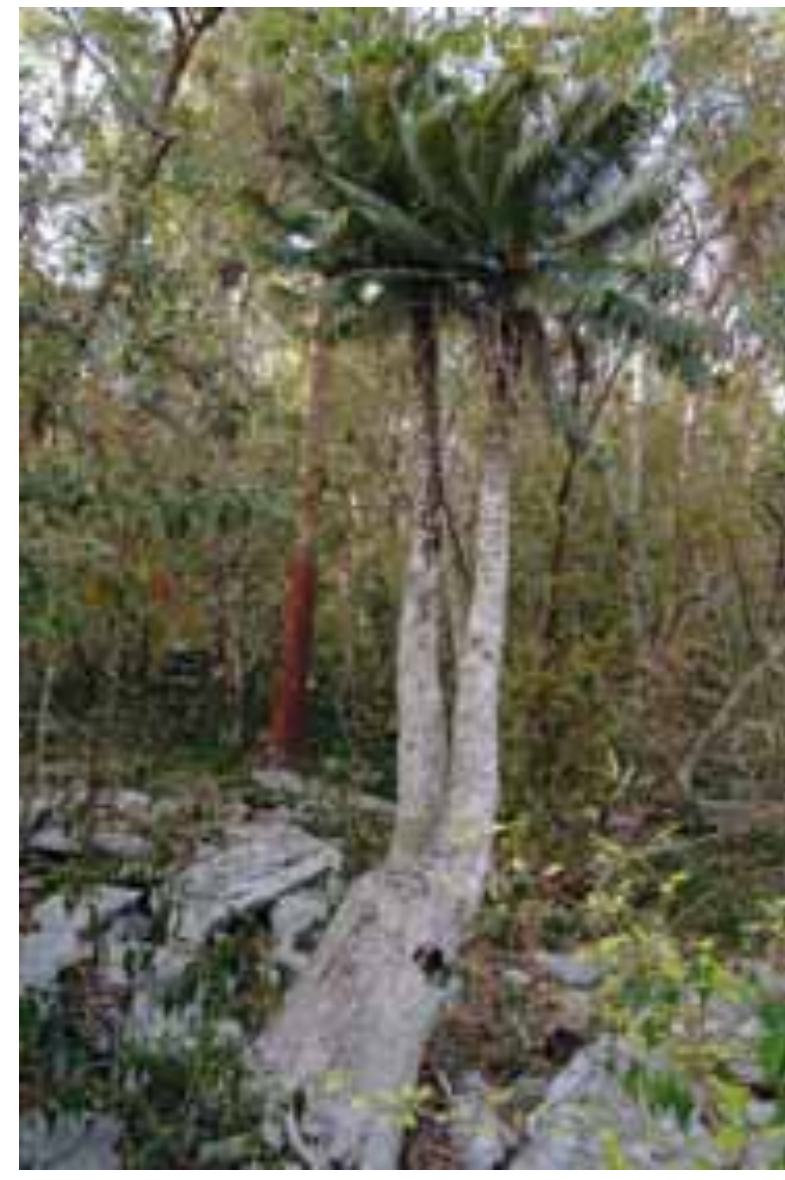

dien noch nicht einwandfrei geklärt werden. Die bisherigen Ergebnisse deuten darauf hin, dass die Cycadeen möglicherweise die Schwestergruppe zu den restlichen Nacktsamern bzw. Koniferen darstellen (Најіваваеi et al. 2006, RAI et al. 2008). Auch innerhalb der Cycadeen bestehen noch offene Fragen bezüglich der 


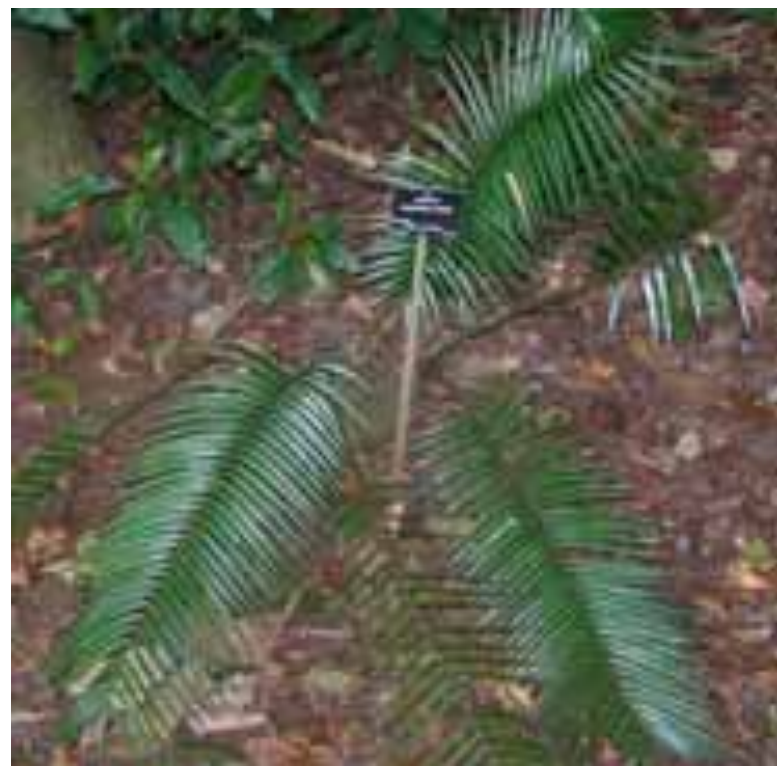

verwandtschaftlichen Beziehungen der einzelnen Gattungen.

In den Cycadeen werden meist die beiden Familien Cycadaceae und Zamiaceae unterschieden, erstere beinhaltet nur die Gattung Cycas, letztere die restlichen Gattungen. Ob die Stangeriaceae mit den beiden Gattungen Stangeria und Bowenia ebenfalls als eigene Familie anerkannt werden sollen, ist umstritten. Aufgrund von morphologischen Daten scheint dies gerechtfertigt (Stevenson 1992, Hermsen et al. 2006), molekulare Daten bieten dafür aber bisher keine ausreichende Unterstützung (z.B. ZGURSKI et al. 2008).

Wie bereits erwähnt, sind die Reproduktionsorgane meist zapfenförmig. Männliche und weibliche Organe finden sich dabei auf getrennten Pflanzen. Die weiblichen Zapfen sind in der Regel weniger zahlreich, dafür aber wesentlich größer. Bis zu $45 \mathrm{~kg}$ kann ein einzelner weiblicher Zapfen auf die Waage bringen. Die Bestäubung erfolgt durch Käfer oder den Wind. Ein zur Zeit der Bestäubung von der Samenanlage ausgeschiedener Bestäubungstropfen erlaubt ein effektives Auffangen der Pollenkörner. Nach mehreren Zellteilungen gehen aus der generativen Zelle schließlich frei bewegliche, d.h. begeißelte Spermatozoiden hervor, eine Eigenschaft, die innerhalb der heutigen Samenpflanzen ansonsten nur noch von Ginkgo her bekannt ist.

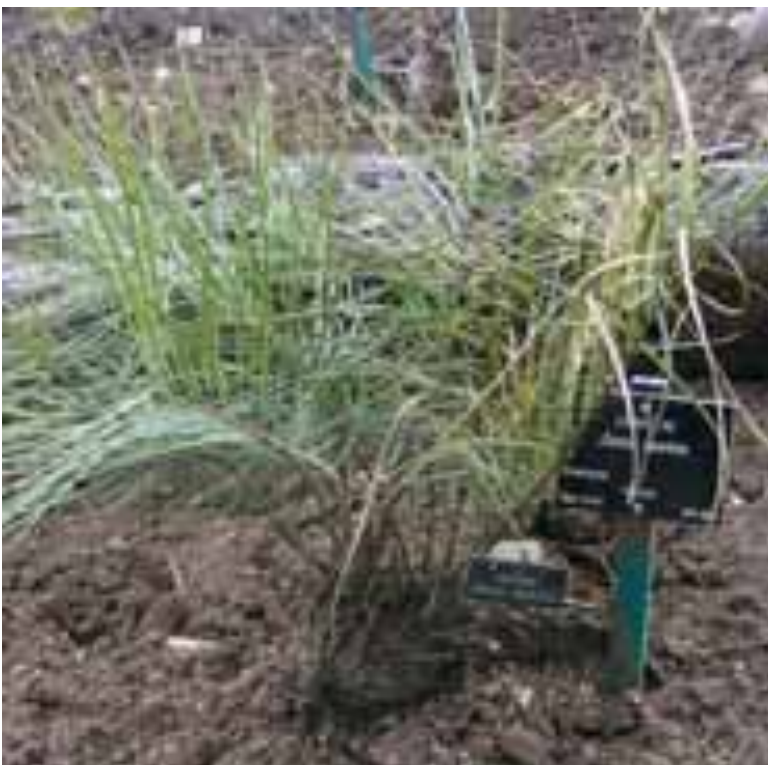

Cycadeen-Samen besitzen meist eine auffällig gefärbte, fleischige Samenschale, die der Anlockung und Ausbreitung durch Tiere dient. Bei Cycas kann diese äußere Schicht auch aus einem schwammartigen Gewebe bestehen, das die Schwimmfähigkeit und Lebensfähigkeit in Salzwasser erhöht. In dieser Eigenschaft liegt vermutlich die vergleichsweise große Verbreitung dieser Gattung begründet (Norstog \& Nicholls 1997).

Mit knapp 300 Arten (Osborne et al. 1999, Hill et al. 2004) sind die Cycadeen nach den Koniferen die artenreichste Gruppe der Gymnospermen. Diese für lebende Fossilien außergewöhnliche Vielfalt spiegelt sich auch in der Vielfalt ihrer über den Globus verteilten Lebensräume wider. Alle Cycadeen-Gattungen sind jeweils auf einen einzigen Kontinent be-

Abb. 2 (oben links): Ceratozamia sabatoi, eine gefährdete Art Zentralmexikos.

Abb. 3 (oben rechts): Zamia spartea, eine seltene, stark bedrohte Art Südmexikos.

Abb. 4 (Seite 7 oben): Dioon edule, eine attraktive Zierpflanze, die in Zentralmexiko beheimatet ist. Die Pflanze wird heutzutage vor allem wegen ihrer attraktiven Blattkrone ,geerntet", früher nutzte man auch ihre essbaren Samen.

Abb. 5 (Seite 7 unten): Palmetum des Botanischen Gartens Xalapa mit der Königspalme, Roystonea regia, im Vordergrund. 
schränkt mit Ausnahme von Cycas, die in Australien, Südostasien und Afrika vorkommt. Encephalartos und Stangeria finden sich nur im kontinentalen Afrika, Bowenia, Macrozamia und Lepidozamia sind Endemiten Australiens, die restlichen Gattungen (Ceratozamia, Chigua, Dioon, Microcycas, Zamia) sind hingegen neuweltlichen Ursprungs. Die Habitate, in denen Cycadeen heimisch sind, unterscheiden sich von Art zu Art sehr. Einige Arten wachsen in tropischen Regenwäldern, andere in wechselfeuchten Wäldern, in denen ausgeprägte Trockenperioden herrschen. Auch sehr trockene Standorte mit seltenen, unregelmäßig auftretenden Regenfällen werden besiedelt, zum Beispiel Savannen.

\section{Mexikanische Cycadeen - \\ Diversität, Nutzung und Gefährdung}

Mexiko gehört mit geschätzten 18000 bis 30000 Pflanzenarten zu den artenreichsten Ländern der Welt (Toledo et al. 1997). Es stellt auch eines der Diversitätszentren der Cycadeen dar und beherbergt innerhalb der Neuen Welt die meisten Arten dieser Gruppe. Allein drei Gattungen und 57 Arten, d. h. etwas mehr als ein Sechstel der weltweiten Artenvielfalt, sind dort zu finden (Hill et al. 2007, NicolaldeMorejón et al. 2011, Vovides, pers. Mitteilung 2011). Am artenreichsten ist die Gattung Ceratozamia (Abb. 2) mit 23 Arten, gefolgt von Zamia (Abb. 3) mit 22 Arten. Die Gattung Dioon weist 12 Arten auf. Die mexikanischen $\mathrm{Cy}$ cadeen sind über das Land verstreut und besiedeln verschiedene Habitate. Ceratozamia und Zamia finden sich in immergrünen Tieflandregenwäldern ebenso wie in kühleren Eichen-Kiefern-Wäldern höherer Lagen, aber auch in Trockenwäldern. Dioon ist generell eher in Trocken- oder Dornwäldern bzw. -gebüsch anzutreffen. Ein Problem vieler Arten ist, dass sie nur sehr kleinräumig auftreten. Dadurch sind sie sehr anfällig für Eingriffe des Menschen, mit der Folge, dass viele Arten stark bedroht sind.

Früher wurden Cycadeen ihrer stärkereichen Samen wegen als Nahrungsquelle genutzt. Auch heute noch finden sich in Mexiko Regionen, in denen Cycadeen-Samen in Zeiten
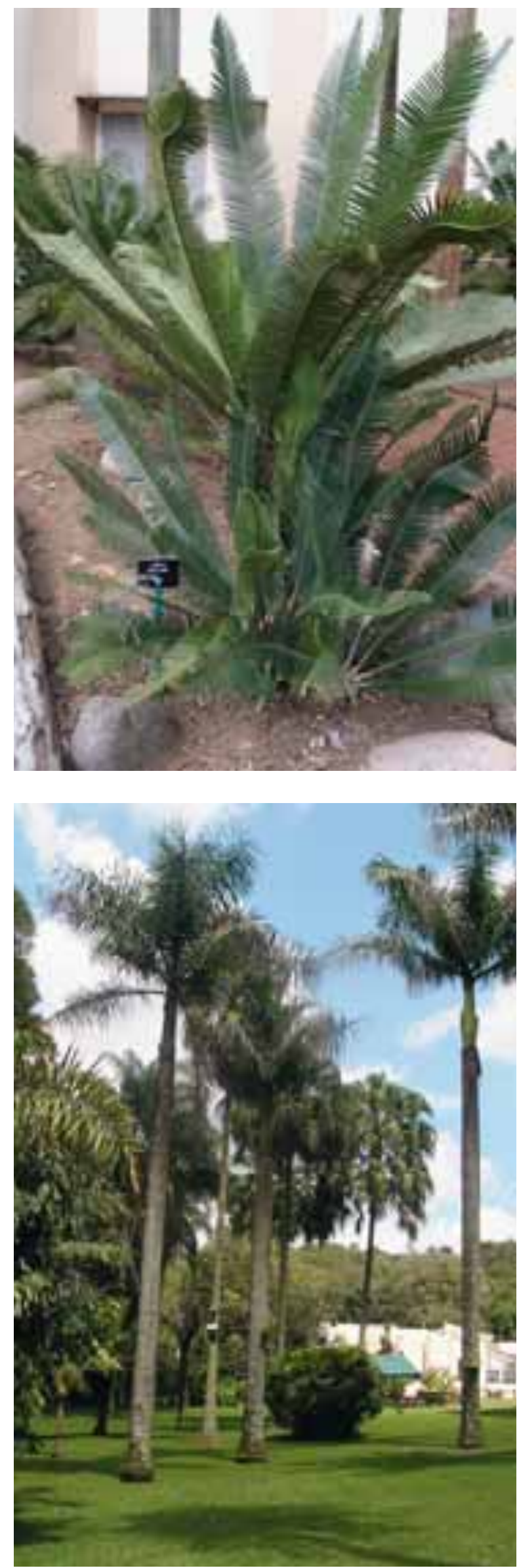


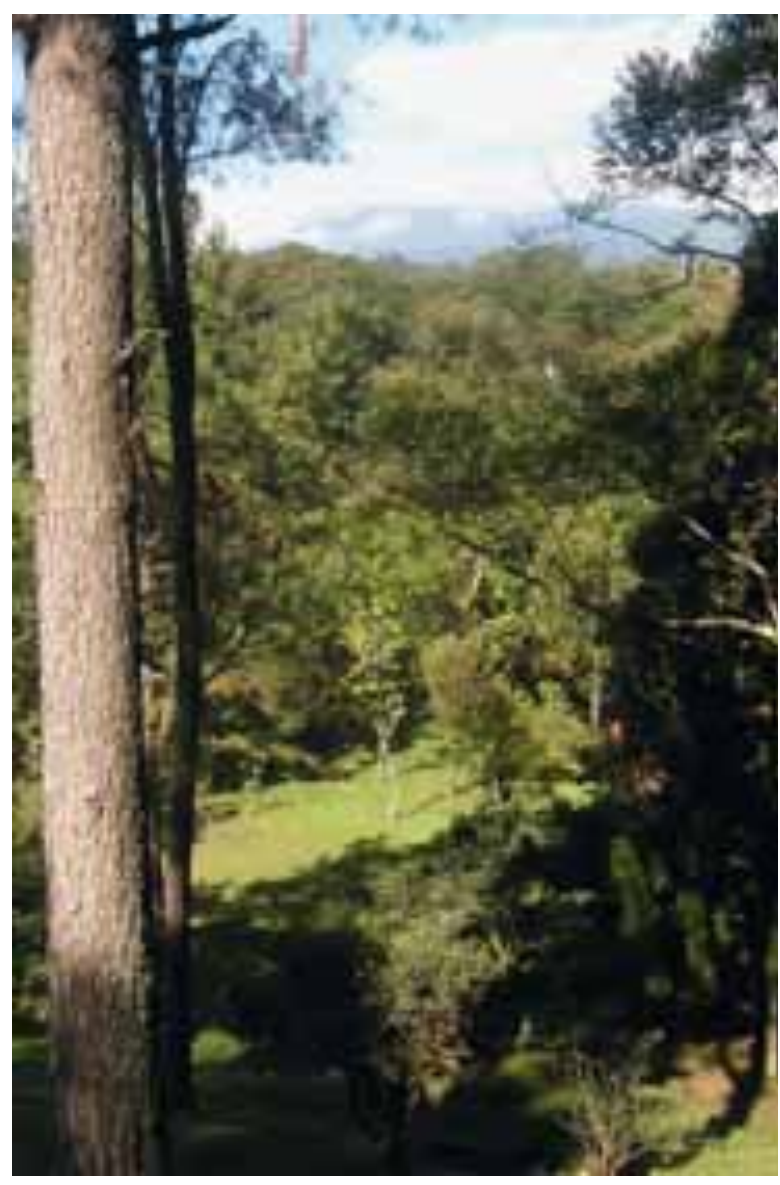

schlechter Maisernten als Ersatznahrungsquelle Verwendung finden. Hiervon geht jedoch im Allgemeinen keine Bedrohung für die Pflanzen aus. Diese resultiert vielmehr aus der Zerstörung der Lebensräume sowie aus anderen Nutzungsformen. Vielfach spielt der illegale Handel eine Rolle, vielerorts werden die Pflanzen aber auch zu Dekorationszwecken genutzt und dadurch geschädigt. Zum Beispiel dienen die Blattkronen von Arten wie Dioon edule (Abb. 4) oder D. mejiae als Grabschmuck oder als Schmuck bei religiösen Festlichkeiten, so zum Beispiel den Palmsonntagfeiern (Haynes \& Bonta 2007, Octavio-Aguilar et al. 2008). Dabei werden in großem Stil Pflanzen „geköpft“. Zwar können sich die Pflanzen gelegentlich innerhalb von 10-15 Jahren regenerieren, doch viele Individuen überleben die Entfernung der Sprossspitze nicht.

Insgesamt sind $62 \%$ aller weltweiten Cycadeen-Arten vom Aussterben bedroht, wodurch sie den unrühmlichen Status der am stärksten gefährdeten Pflanzengruppe der Welt haben
(IUCN 2010). Allein $88 \%$ der Zamiaceen gelten nach der globalen Roten Liste 1997 als gefährdet (Walter \& Gillett 1998). Alle Cycadeen befinden sich zudem in den Anhängen I oder II des Washingtoner Artenschutzabkommens (CITES), so dass der internationale Handel mit diesen Arten eingeschränkt bzw. vollständig untersagt ist. Mexiko ist hinsichtlich seiner Cycadeen-Flora insofern von großer Bedeutung, als dass mehr als 80 Prozent der mexikanischen Cycadeen-Arten dort endemisch sind (Pérez-Farrera \& Vovides 2006). Daher sind die menschlichen Eingriffe dort besonders gravierend für den Gesamtbestand an Cycadeen. Zurzeit werden 35 Arten der Cycadeen Mexikos als gefährdet bzw. stark gefährdet eingestuft (IUCN 2010), Tendenz steigend.

\section{Schutzprojekte und die Rolle des Botanischen Gartens Xalapa}

Xalapa ist die Hauptstadt des mexikanischen Bundesstaates Veracruz und liegt an der Ostabdachung der Sierra Madre Oriental, einer der Hauptgebirgszüge Mexikos, in einer Höhe von knapp 1300 m ü.d.M. In dieser Höhenlage befinden sich natürlicherweise durch hohe Niederschläge und gemäßigte Temperaturen gekennzeichnete artenreiche Nebelwälder, die jedoch in weiten Teilen durch menschlichen Einfluss zerstört oder in ihrem Bestand verändert wurden. Insbesondere der Kaffeeanbau spielt hierbei eine große Rolle. Einer der wenigen noch weitgehend ungestörten Nebelwälder in der Umgebung von Xalapa lässt sich im Botanischen Garten der Stadt beobachten, insbesondere in dem direkt an den Schaugarten angrenzenden Schutzgebiet.

Der Botanische Garten von Xalapa ist aber nicht nur ein Refugium für bedrohte Nebel-

Abb. 6 (oben): Blick vom Pinetum über den Botanischen Garten Xalapa.

Abb. 7 (Seite 9 links): Quercus xalapensis, eine lokale Eichenart.

Abb. 8 (Seite 9 rechts): Marattia laxa, eine stark bedrohte Farnart der mexikanischen Nebelwälder. Die Detailaufnahme (im Bild links) zeigt die Sori. 


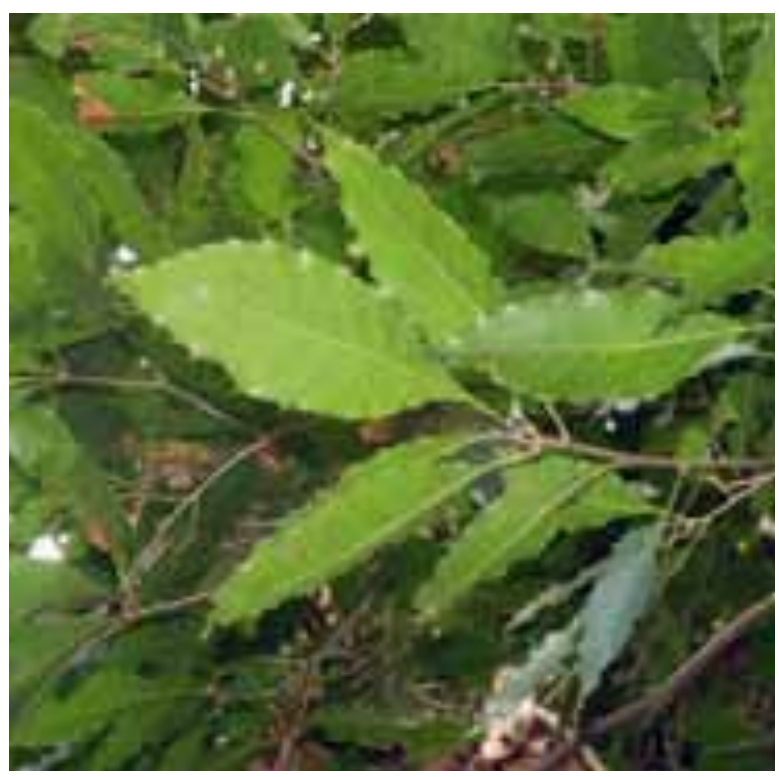

wälder, sondern auch für viele in Mexiko bedrohte Arten, allen voran die mexikanischen Cycadeen.

Der Botanische Garten wurde 1977 gegründet und unter der fachlichen Anleitung der Royal Botanic Gardens, Kew, England, gestaltet. Er umfasst verschiedene thematische Bereiche. Besonders auffällig ist das zentral gelegene Palmetum (Abb. 5), das größtenteils aus exotischen Palmenarten besteht, aber auch einzelne einheimische Arten wie Sabal mexicana oder Astrocaryum mexicanum zeigt. Ein kleiner Bereich ist den Nutz- und Zierpflanzen gewidmet und in Beetform angelegt. Das Arboretum ist eine lose Anordnung von fremdländischen und einheimischen Gehölzen. Manche der einheimischen Arten sind stark bedroht und Bestandteil einer der nationalen Schutzsammlungen des Gartens, so zum Beispiel die beiden Magnoliaceen Magnolia dealbata und Talauma mexicana. Eichen (Quercus spp.) und Kiefern (Pinus spp.) sind charakteristische Elemente der mexikanischen Bergwälder und daher besitzt der Garten auch ein Pinetum (Abb. 6), in dem sich Arten wie Pinus teocote und $P$. ayacahuite neben anderen Gymnospermen wie Podocarpus guatemalensis und der Zypressenart Cupressus benthamii befinden. Eichenarten finden sich wiederum im angrenzenden Nebelwald, insbesondere die natürlicherweise lokal vorkommende Quercus $x a$ lapensis, die Xalapa-Eiche (Abb. 7). Der Nebel-

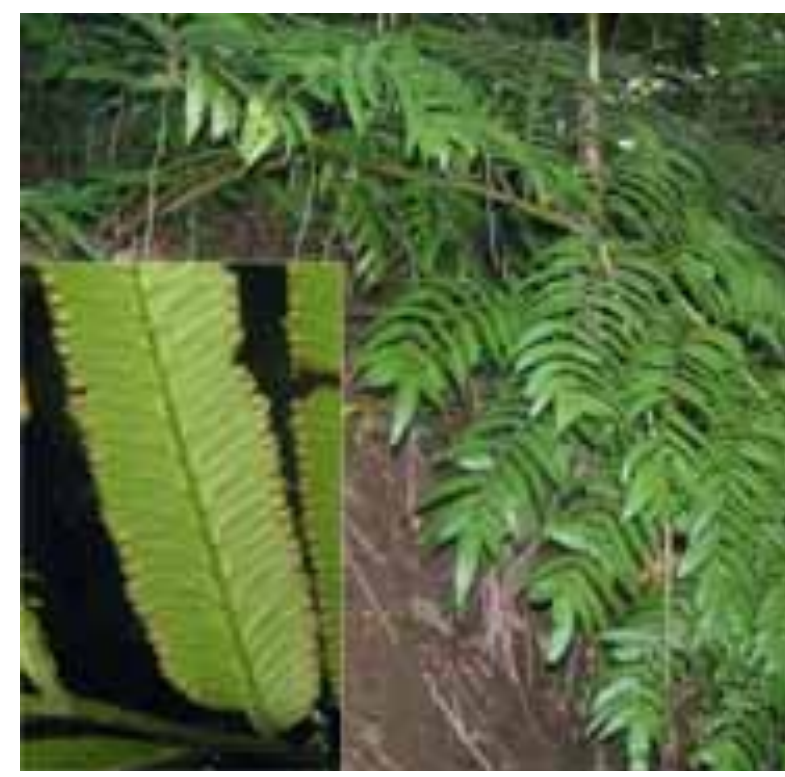

wald ist der einzige naturbelassene Bereich des Botanischen Gartens und besteht neben den Eichen unter anderem aus Vertretern der Gattungen Carpinus, Clethra, Liquidambar oder Ostrya, die eher Elemente der gemäßigten Breiten sind. Tropische Elemente sind zum Beispiel die Gattungen Cedrela, Meliosma oder Oreopanax. Der Nebelwald des Gartens wurde in den letzten Jahren auch verstärkt für die natürliche Regeneration bedrohter Arten wie den Farn Marattia laxa (Abb. 8) genutzt.

Den sicherlich bedeutendsten Teil des Gartens stellt allerdings die Nationale Schutzsammlung mexikanischer Cycadeen dar (Abb. 9). Aktuell umfasst sie 54 der 57 bekannten Arten Mexikos und 1652 Pflanzen, einschließlich der aus künstlicher Bestäubung hervorgegangenen Jungpflanzen (Vovides, pers. Mitteilung 2011). Hinzu treten noch mindestens je ein Vertreter der nicht in Mexiko beheimateten Cycadeen-Gattungen, so dass es sich um eine der weltweit wichtigsten Sammlungen dieser Pflanzengruppe handelt. Ziel der Schutzsammlung ist jedoch nicht nur die ex-situ-Erhaltung der Arten in Botanischen Gärten, sondern auch die Ausbringung der kultivierten Individuen in situ, d. h. an den Wildstandorten. Seit den 90er Jahren hat es mehrere solcher Wiederansiedlungsprojekte durch den Botanischen Garten Xalapa in Biosphärenreservaten in den Bundesstaaten Chiapas und Puebla gegeben, unter an- 


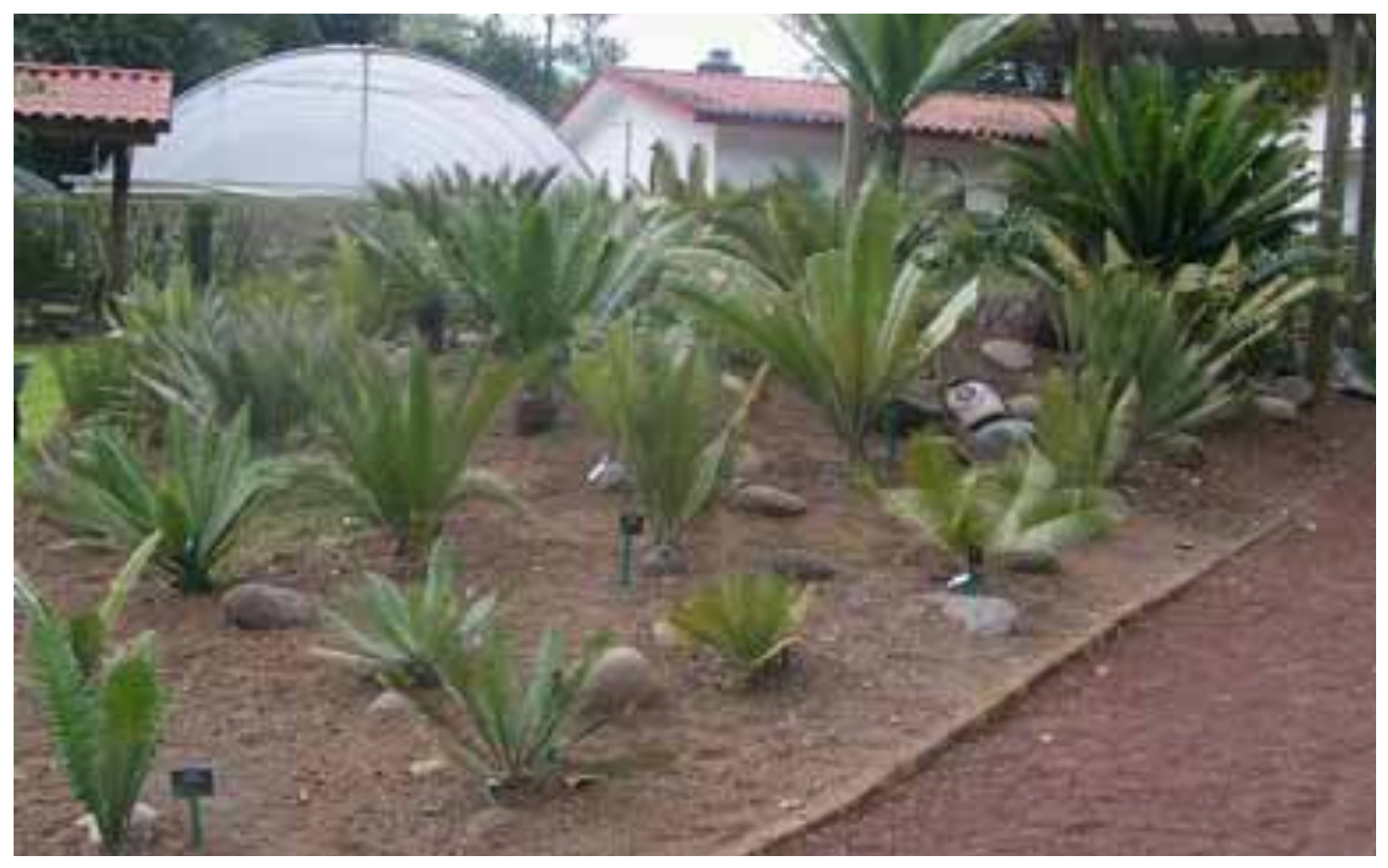

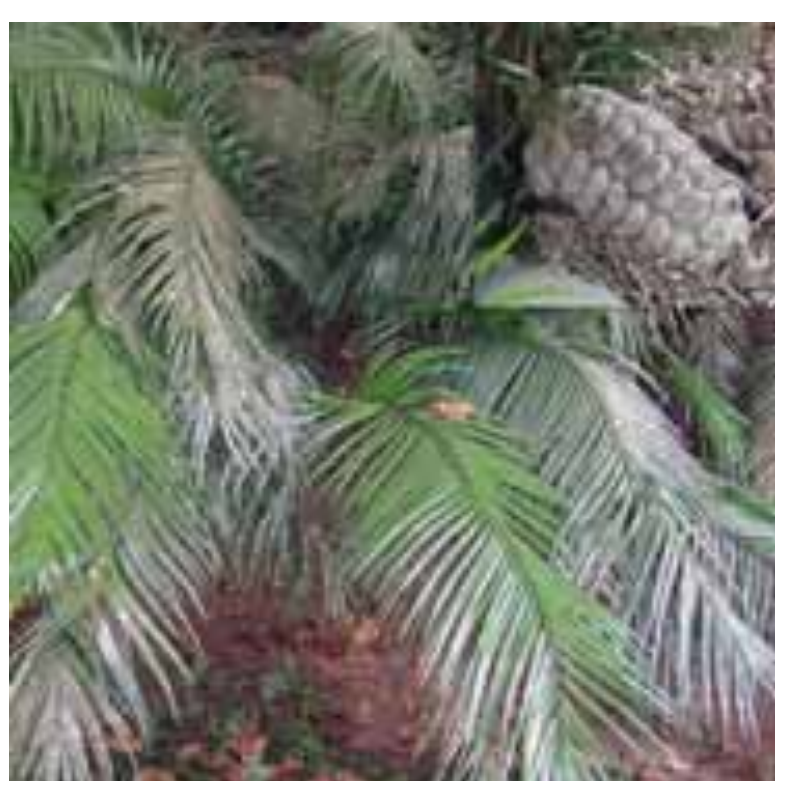

derem für die Arten Ceratozamia mirandae, C. matudae, Zamia soconuscensis (Abb. 10), Dioon merolae (PÉREZ-FARRERA et al. 1999, Pérez-Farrera et al. 2000, Vovides et al. 2002) und kürzlich auch für die vom Aussterben bedrohte D. caputoi (Vovides et al. 2006). Ebenso konnten Bauern mancherorts vom Vorteil des Schutzes der Arten an den Wildstandorten überzeugt und gleichzeitig gewonnen werden, Pflanzen-Anzuchten für die Kultivierung der lokal vorkommenden Cycadeen-Arten aufzubauen. Dies hat an den betroffenen Orten in erheblichem Maße dazu beigetragen, den Druck auf die Cycadeen-Bestände zu nehmen. Trotz anfänglicher Schwierigkeiten haben sich die meist auf Gemeinde-Ebene organisierten, offiziell registrierten Baumschulen positiv entwickelt und bringen den beteiligten Bauern ein alternatives Zusatzeinkommen über den regulierten Verkauf der Pflanzen.

Auch wenn es sich hierbei bisher nur um den berühmten Tropfen auf den heißen Stein handelt, bleibt die Hoffnung, dass die genannten Projekte weiter Schule machen und so zu einer umfassenderen Stabilisierung oder auch Erholung der Cycadeen-Populationen in absehbarer Zukunft beitragen. Vielleicht gelingt es sogar, für die eine oder andere Art einen positiveren Gefährdungsstatus in den Roten Listen zu erzielen.

Abb. 9 (oben): Der Cycadeengarten bildet eine nationale Schutzsammlung und beherbergt 54 der 57 mexikanischen Taxa sowie Vertreter aller weltweit vorkommenden Gattungen.

Abb. 10 (unten): Zamia soconuscensis, eine Art der Soconusco-Berge im Süden Mexicos. An der Basis befindet sich ein Zapfen (im Bild oben rechts). 


\section{Dank}

Der Dank gilt dem Instituto de Ecología, Xalapa, Mexico, insbesondere Miguel Rubio GoDoY, dessen Einladung dem Erstautor den Besuch des Botanischen Gartens erlaubt hat; außerdem Andrew P. Vovides, einem der wissenschaftlichen Betreuer der Cycadeen-Schutzsammlung des Botanischen Garten Xalapa, für Informationen über den aktuellen CycadeenBestand. Georg Zizka danken wir für das Überlassen von Bildmaterial.

\section{Literatur}

Brenner, E.D., Stevenson, D.W. \& Twigg, R.W. 2003:

Cycads: Evolutionary innovations and the role of plant-derived neurotoxins. - Trends Pl. Sci. 8: 446-452. Donaldson, J.S. (Hrsg.) 2003: Cycads. Status survey and conservation action plan. IUCN/SSC Cycad specialist group. IUCN, Gland, Schweiz und Cambridge, UK. Donaldson, J.S., Hill, K.D. \& Stevenson, D.W. 2003: Cycads of the world: an overview. In: Donaldson, J. (Hrsg.), Cycads. Status survey and conservation action plan. IUCN/SSC Cycad Specialist Group, IUCN, Gland, Switzerland and Cambridge, UK, S. 3-19.

GaO, Z-F. \& Thomas, B.A. 1989: A review of fossil cycad megasporophylls, with new evidence of Crossozamia Pomel and its associated leaves from the lower Permian of Taiyuan, China. Rev. Palaeobot. Palynol. 60: 205-223. Hajibabaei, M., Xia , J. \& Drouin, G. 2006: Seed plant phylogeny: Gnetophytes are derived conifers and a sister group to Pinaceae. Mol. Phylogenet. Evol. 40: 208-217. Haynes, J.L. \& Bonta, M.A. 2007: An emended description of Dioon mejiae Standl. \& L.O. Williams (Zamiaceae). Proceedings of the 7th International Conference on Cycad Biology, Xalapa, Mexico, January 2005. Mem. New York Bot. Gard. 97: 418-443.

Hermsen, E.J., Taylor, T.N., TAylor, E.L. \& Stevenson, D.W. 2006: Cataphylls of the middle Triassic cycad Antarcticycas schopfii and new insights into cycad evolution. Amer. J. Bot. 93: 724-738.

Hill, K., Stevenson, D.W. \& Osborne, R. 2004: The world list of cycads. In: Walters, T. \& Osborne, R. (Hrsg.) Cycad classification: concepts and recommendations. - Massachusetts, S. 219-235.

Hill, K.D., Stevenson, D.W. \& Osborne, R. 2007: The world list of cycads. In: Proceedings of the 7th International Conference on Cycad Biology (CYCAD 2005), Xalapa, Mexico, January 2005. Mem. New York Bot. Gard. 97: 454-483.

IUCN 2010: The IUCN Red List of threatened species, October 2010 update: Cycads facts. IUCN, Gland. Online: http://cmsdata.iucn.org/downloads/cycad_ factsheet_final.pdf.

Jones, D.L. 2002: Cycads of the world: ancient plants in today's landscape. 2. ed. - Sydney.

Loran, M. 2002: In: Whitelock, L. M.: The Cycads. - Portland.
Nicolalde-Morejón, F., Vergara-Silva, F., GonzálezAstorga, J., Stevenson, D.W., Vovides, A.P. \& Sosa, V. 2011: A character-based approach in the Mexican cycads supports diverse multigene combinations for DNA barcoding. Cladistics 27: 150-164.

Norstog, K. J. \& Nicholls, T.J. 1997: The biology of cycads. - Ithaca, New York.

Octavio-Aguilar, P., GonzÁlez-Astorga, J. \& Vovides, A.P. 2008: Population dynamics of the Mexican cycad Dioon edule Lindu. (Zamiaceae): life history stages and management impact. Bot. J. Linn. Soc. 157: 381-391. Osborne, R., Stevenson, D.W. \& Hill, K. 1999: The world list of cycads. In: Chen, C.J. (Hrsg.), Biology and conservation of Cycads. Fourth International Conference on Cycad Biology. - Beijing, S. 224-239.

Pérez-Farrera, M.A., Quintana-Ascencio, P.F., Salvatierra-Izaba, B. \& Vovides, A.P. 2000: Population dynamics of Ceratozamia matudae Lundell (Zamiaceae) in El Triunfo Biosphere Reserve, Chiapas, Mexico. - Bull. Torrey Bot. Club 127: 291-299.

Pérez-Farrera, M.A., Vovides, A.P. \& AlvarezMoctezuma, J.G. 1999: A study on seed germination of the cycad, Dioon merolae (Zamiaceae). New Plantsman 6: 214-218.

Rai, H.S., Reeves, P.A., Peakall, R., Olmstead, R.G. \& Graham, S.W. 2008: Inference of higher-order conifer relationships from a multi-locus plastid data set. Botany 86: 658-669.

Stevenson, D.W. 1992: A formal classification of the extant cycads. Brittonia 44: 220-223.

Toledo, V. M., Rzedowski, J. \& Villa-Lobos, J. 1997 : Mexico - regional overview. In: Davis, S.D., HeYwood, V.H., Herrera-MacBryde, O., Villa-Lobos, J. \& Hamilton, A. (Hrsg.) 1997: Centres of plant diversity: A guide and strategy for their conservation. Volume 3: The Americas. IUCN Publications Unit, Cambridge, England. Vovides, A. P., Cortéz, M. E., Iglesias, C. G. \& Lascurain, M. 1995: The Jardin Botánico Francisco Javier Clavijero in Xalapa, Veracruz, Mexico. BGCNews 2(5). Vovides, A. P. \& Pérez-Farrera, M.A. 2006: The ceremonial use of the threatened "Espadaña" cycad (Dioon merolae, Zamiaceae) by a community of the central depression of Chiapas, Mexico. Bol. Soc. Bot. Mex. 78: 107-113.

Vovides, A. P., Pérez-Farrera, M.A. \& Iglesias, C. 2006: Sixteen years of cycad propagation in rural nurseries in Mexico: An alternative conservation strategy aimed at sustainable management. The Nature of Success: Success for Nature. Proceedings of the $6^{\text {th }}$ BGCI international congress on Education in Botanic Gardens, September 2006, Oxford, U.K.

Walter, K.S. \& Gillett, H.J. (Hrsg.) 1998: 1997 IUCN Red List of Threatened Plants. Compiled by the World Conservation Monitoring Centre. IUCN - The World Conservation Union, Gland, Schweiz.

Zgurski, J.M., Rai, H.S., FaI, Q.M., Bogler, D.J., Francisco-Ortega, J. \& Graham, S.W. 2008: How well do we understand the overall backbone of cycad phylogeny? New insights from a large, multigene plastid data set. Mol. Phylogenet. Evol. 47: 1232-1237. 